\title{
Meningitis caused by an alkali-producing pseudomonad
}

\author{
W. A. COWLISHAW, MARGARET E. HUGHES, AND H. C. R. SIMPSON \\ From the Department of Microbiology, General Hospital and Children's Hospital, Nottingham
}

SYNOPSIS The clinical and microbiological features of a case of meningitis, due to an alkaliproducing pseudomonad which closely resembles Pseudomonas pseudoalcaligenes, are described. A respiratory infection and a course of antibiotic therapy before admission to hospital may have been predisposing factors to opportunistic infection by this normally saprophytic organism. The problems of identifying alkali-producing pseudomonads are discussed.

Meningitis in early childhood is commonly caused by Haemophilus influenzae, Neisseria meningitidis, and Streptococcus pneumoniae. Infection by opportunistic bacteria, such as Pseudomonas spp, however, is relatively uncommon. We present this as the first isolation of Pseudomonas pseudoalcaligenes from cerebrospinal fluid (CSF) and as the first instance of its pathogenicity. Ps. pseudoalcaligenes was first described by Stanier et al (1966). Pedersen et al (1970) and Gilardi (1972) reported the recovery of this organism from many clinical specimens but in none of these instances was it considered to be pathogenic.

\section{Case report}

An 8-month-old female infant was admitted to hospital with a 24-hour history of anorexia, vomiting, drowsiness, and irritability. There had been no diarrhoea.

After contracting whooping cough at $4 \frac{1}{2}$ months, she developed a chronic cough. This persisted until the time of admission, despite a 14-day course of ampicillin, $125 \mathrm{mg}$ four times daily, from her general practitioner, ending three days previously. Her birth history and development had been normal.

On admission her skin was ashen, with a sparse macular purpuric rash of patchy distribution, $0.5 \mathrm{~cm}$ in diameter, on the trunk, upper limbs, and feet. This was not typical of the maculopapular erythema found in ampicillin sensitivity. The spleen was not palpable. Her temperature was $38 \cdot 6^{\circ} \mathrm{C}$ and pulse 180/

Received for publication 7 April 1976 minute; the anterior fontanelle was tense and her throat was reddened. Occasional twitches of the limbs were noted. There was no neck stiffness.

Results of laboratory investigations were as follows: haemoglobin $10.7 \mathrm{~g} / \mathrm{dl}$; leucocytes $6500 / \mu 9$ with $60 \%$ neutrophils, $36 \%$ lymphocytes and $4 \%$. monocytes; platelet count $163000 / \mu \mathrm{l}$; ESR $36 \mathrm{mf}$ fall in 1 hour (Westergren). Blood cultures were sterile after 10 days' incubation, urine was sterile, and a throat swab grew only commensal organisms. The CSF was opalescent with a leucocyte count of $\stackrel{\mathbb{2}}{\Omega}$ $400 / \mu$ l, of which $90 \%$ were neutrophils and $10 \% \overrightarrow{\overrightarrow{0}}$ lymphocytes. Protein concentration was $2.1 \mathrm{~g} / 1$ and 3 sugar $0.55 \mathrm{mmol} / 1$. Numerous pleomorphic Gramnegative bacilli with distorted cell walls, indicative of antibacterial activity, were seen in a stained film from a centrifuged deposit of CSF. An organism was grown which was later shown to conform closely to Ps. pseudoalcaligenes. Leucocyte counts in further specimens of CSF, taken 24 and 48 hours later, were 1225 and $1200 / \mu$ l respectively. Although stained films from both of these specimens showed scanty Gram-negative bacilli, cultures were sterile.

In view of the laboratory findings, initial antibiotic therapy was with ampicillin, $400 \mathrm{mg} / \mathrm{kg}$ per day, $N$ intravenously. After 24 hours this was changed, on $N$ the basis of the in vitro sensitivity of the organism $N$ recovered from the CSF, to $6 \mathrm{mg} / \mathrm{kg}$ per day of $\omega$ gentamicin intravenously and $2 \mathrm{mg}$ daily intrathecally for seven days. Chloramphenicol, $100 \mathrm{mg} / \mathrm{kg}$ per day, was then given orally for a further seven days. $\stackrel{\Phi}{\rightarrow}$

The condition of the patient was unchanged until 24 hours after starting gentamicin, but subsequently she made an uncomplicated recovery. One month later her head circumference had not increased, 088 
hearing and vision appeared normal, and she did not seem to have suffered any regression in development.

\section{BACTERIOLOGY}

After 18 hours at $37^{\circ} \mathrm{C}$, cultures yielded a moderate growth of a non-pigmented Gram-negative bacillus which grew well aerobically on blood agar and MacConkey agar, and on chocolate agar in an atmosphere of $10 \% \mathrm{CO}_{2}$.

Of the preliminary tests carried out, the following results showed that the organism was an alkaliproducing pseudomonad: production of cytochrome oxidase, motile with one polar flagellum (Kirkpatrick's stain), and production of alkali in the Hugh and Leifson O-F test. The results of secondary tests (Stanier et al, 1966), which follow, enabled the organism to be provisionally identified as $P S$. pseudoalcaligenes: nitrates were reduced to nitrites, growth was absent at $4^{\circ} \mathrm{C}$ and present at $41^{\circ} \mathrm{C}$, gelatin was not liquefied, and starch was not hydrolysed.

The organism was sent to the National Collection of Type Cultures (Computer Trials Laboratory), where it was confirmed to resemble closely $P s$. pseudoalcaligenes differing from the type strain of $P s$. pseudoalcaligenes (NCTC 10860) by only two results of a total of 87 tests. These differences are listed as follows:

\section{Test}

NCTC Strain from 10860 CSF

Acid from glycerol

(ammonium salt medium) $\quad-\quad+$

Growth on $\beta$-hydroxybutyrate $+{ }_{-*}^{*}$

*Accumulation of poly $\beta$-hydroxybutyrate inclusion granules -positive when organism grown on nutrient agar containing $\beta$-hydroxybutyrate.

Antibiotic sensitivity tests were carried out in our laboratory by the disc diffusion method on Oxoid Diagnostic Sensitivity Test agar with the addition of $5 \%$ lysed horse blood. The pseudomonad was found to be sensitive to the following antibiotics (with the antibiotic content of each disc in parenthesis): tetracycline $(10 \mu \mathrm{g})$, chloramphenicol $(50 \mu \mathrm{g})$, carbenicillin $(100 \mu \mathrm{g})$, cephaloridine $(30 \mu \mathrm{g})$, cotrimoxazole $(25 \mu \mathrm{g})$, colistin $(10 \mu \mathrm{g})$, gentamicin $(10$ $\mu \mathrm{g})$, neomycin $(30 \mu \mathrm{g})$, sulphafurazole $(100 \mu \mathrm{g})$, and streptomycin $(25 \mu \mathrm{g})$; and resistant to ampicillin $(25$ $\mu \mathrm{g})$ and cephalexin $(30 \mu \mathrm{g})$. The minimum inhibitory concentration of ampicillin, determined by the plate dilution method, was $60 \mu \mathrm{g} / \mathrm{ml}$ using an inoculum of $10^{4}$ organisms $/ \mathrm{ml}$.

\section{Discussion}

Stanier et al (1966) examined several pseudomonads which they recognized as strains of a new species, $P$ s. pseudoalcaligenes. Among these was an isolate from a sinus discharge (ATCC 17440, NCTC 10860). Of 54 isolations of Ps. pseudoalcaligenes reported by Pedersen et al (1970), 32 were from sputum, four from urine, and the remainder from the hospital environment. Gilardi (1972) reported one isolation from a postoperative infection of the knee and another from the blood and sputum of a patient with pneumonia. In none of these instances was Ps. pseudoalcaligenes considered to be pathogenic.

The alkali-producing pseudomonads are difficult to recognize and in a routine laboratory oxidasepositive, Gram-negative bacilli which produce alkali in the Hugh and Leifson O-F test are often identified as Alcaligenes spp. Indeed Gilardi (1972) and von Graevenitz (1973) consider that previously reported pathogenic strains of Alcaligenes spp. may have been alkali-producing pseudomonads.

Although Snell (1973) does not include flagellar arrangement in his identification scheme, flagellar stains are of value in separating Alcaligenes spp from pseudomonads (Sutter, 1968; von Graevenitz, 1973); Alcaligenes spp have peritrichous flagella whereas those of pseudomonads are polar.

Separation into species within the group of alkaliproducing pseudomonads presents problems to taxonomists, as this group is biochemically unreactive in conventional tests. The five members in the group, Ps. acidovorans (den Dooren de Jong, 1926), Ps. alcaligenes (Monias, 1928), Ps. diminuta (Leifson and Hugh, 1954), Ps. testosteroni (Marcus and Talalay, 1956), and Ps. pseudoalcaligenes (Stanier et $a l, 1966)$, were first characterized by their utilization of a wide range of carbon compounds as sole energy source. Identification schemes have been devised by Stanier et al (1966), Pedersen et al (1970), and Snell (1973) but carbon source utilization tests are difficult to carry out in a routine laboratory. Until more conventional tests can be used the identification of the alkali-producing pseudomonads will remain a problem.

The antibiotic sensitivities of previous isolates of Ps. pseudoalcaligenes have been discussed by Pedersen et al (1970), who examined 28 strains, all of which were sensitive to chloramphenicol, $96 \%$ sensitive to erythromycin and tetracycline, and $50 \%$ sensitive to ampicillin. Of 16 strains examined by Gilardi (1972), most were found to be sensitive to tetracycline, neomycin, kanamycin, polymyxin, and gentamicin; only $6 \%$ were reported to be sensitive to ampicillin.

In this case the organism was recovered only from 
the initial CSF, but Gram-negative bacilli were seen in stained films in specimens taken 24 and 48 hours later. High dosage may have caused an ampicillin level sufficient to sterilize the CSF in 24 hours, despite the relative insensitivity of the bacilli. McCracken (1972) reported an ampicillin level of 59 $\mu \mathrm{g} / \mathrm{ml}$ in the CSF of a neonate two and threequarter hours after receiving a daily intravenous dose of $250 \mathrm{mg} / \mathrm{kg}$.

Although Ps. pseudoalcaligenes has not previously been reported as causing meningitis, Patrick et al (1975) reported a case of meningitis caused by $P s$. maltophilia in a 70-year-old man with no history of recent antibiotic therapy and no previous debility apart from chronic bronchitis. The respiratory tract was considered to be the most likely route of infection but there was no supporting clinical and laboratory evidence. The present case differs in that an antibiotic had been given in the previous fortnight, specifically for a chest infection. The patient was therefore a candidate for opportunistic infection by reason of her age, repeated chest infections following whooping cough, and a recent course of a broadspectrum antibiotic. We consider that the respiratory tract was the most likely route of infection in this case.

The initial diagnosis was complicated by a purpuric rash, suggesting meningococcal infection, and by the presence of pleomorphic Gram-negative rods resembling Haemophilus influenzae. Ampicillin was given until in vitro antibiotic sensitivity tests showed the organism, later identified as an unusual pseudomonad, to be resistant.

This stresses the importance of isolating the causative organism in meningitis, and the possibility of this being an opportunistic pathogen should be borne in mind if predisposing factors exist.
We are most grateful to Dr H. M. Rice, Department of Microbiology, Nottingham General Hospital for $\rightleftharpoons$ helpful advice in the preparation of this paper, and to $\frac{5}{9}$ Mr B. Holmes, National Collection of Type Cultures, Central Public Health Laboratory, Colindale, London, for characterizing the organism in detail for us.

\section{References}

den Dooren de Jong, L. E. (1926). Bijdrage tot de kennis van het mineralisatieproces. Thesis. Technische Hoogeschool, Delft.

Gilardi, G. L. (1972). Infrequently encountered Pseudomonas $\overline{0}$ species causing infection in humans. Ann. intern. Med., 77, i 211-215.

von Graevenitz, A. (1973). Clinical microbiology of unusual $\vec{N}$ Pseudomonas species. Progr. clin. Path., 5, 185-218.

Leifson, E. and Hugh, R. (1954). A new type of polar monotrichous flagellation. J. gen. Microbiol., 10, 68-70.

McCracken, G. H., Jr. (1972). The rate of bacteriologic 으 response to antimicrobial therapy in neonatal meningitis. Amer. J. Dis. Child., 123, 547-553.

Marcus, P. I. and Talalay, P.(1956). Induction and purification of $\alpha$ - and $\beta$-hydroxysteroid dehydrogenases. J.biol. Chem., 218, 661-674.

Monias, B. L. (1928). Classification of Bacterium alcaligenes pyocyaneum and fluorescens. J. infect. Dis., 43, 330-334

Patrick, S., Hindmarch, J. M., Hague, R. V., and Harris, B $\vec{c}$ M. (1975). Meningitis caused by Pseudomonas maltophilig J. clin. Path., 28, 741-743.

Pedersen, M. M., Marso, E., and Pickett, M. J. (197 Nonfermentative bacilli associated with man. III. Path genicity and antibiotic susceptibility. Amer. J. clin. Path. 54, 178-192.

Snell, J. J. S. (1973). The Distribution and Identification of Non-fermenting Bacteria. (Public Health Laboratory Service Monographs, No. 4.) HMSO, London.

Stanier, R. Y., Palleroni, N. J., and Doudoroff, M. (1966). The aerobic pseudomonads: a taxonomic study. J. gen. Microbiol., 43, 159-271.

Sutter, V. L. (1968). Identification of Pseudomonas species isolated from hospital environment and human sources. Appl. Microbiol., 16, 1532-1538. 